\title{
Sieben Hebel zur Optimierung
}

\author{
Martin Gössler
}

Beratung und Supervision kosten Zeit und Geld. Der Einsatz der richtigen Berater kann beides sparen helfen und den Erfolg der Organisation erhöhen.

Supervision ist in vielen Non-Profit-Organisationen State of the Art. Coaching als spezielles Angebot für Führungskräfte ist vielerorts hinzugekommen. Beachtliche Budgets werden für externe Berater aufgewendet. Doch auch Zweifel wachsen: »Führt Supervision wirklich zu Verbesserungen für unsere Kunden?«»Haben wir gut ausgewählte Berater? «In einer Studie kommen kritische Ergebnisse zutage. So geben beispielsweise in einer psychiatrischen Klinik 24 Prozent der Supervisionsteilnehmer an, dass Supervision keinen oder nur geringen Nutzen hat (Petzold u. a. 2003: Supervision auf dem Prüfstand. Leske und Budrich). Ein bedenkliches Ergebnis.

Die Basisthese dieses Artikels: Viele Organisationen schöpfen das volle Potenzial eines effektiven Einsatzes von Supervision nicht aus. Und tun zu wenig, um Risiken und unerwünschte Nebenwirkungen zu minimieren. Im Folgenden werden sieben wirksame Hebel vorgestellt, die den Einsatz von Supervision verbessern können. Das Gesagte gilt auch — mit kleinen Abstrichen oder Adaptionen - für verwandte Beratungsleistungen wie Coaching, Teamentwicklung oder Organisationsentwicklung.

\section{Hebel 1: Für klare Zielsetzung sorgen}

»Wir haben zwar eine Lösung, aber wissen wir eigentlich für welches Problem? «So kommentierte eine Personalentwicklerin den Einsatz von Supervision in ihrer Organisation. Je klarer es gelingt, Zielsetzungen der Beratung herauszuarbeiten, desto höher wird der Nutzen sein. Entlang der Leitfrage: »Was sind die wichtigsten Herausforderungen in den nächsten Jahren?« kann sich ein gänzlich unterschiedlicher Einsatz von Supervision ergeben. Potenziell kann Supervision bei folgenden fünf Themen Nutzen stiften:

- Qualitätssicherung: Optimierung der fachlichen Arbeit

- Konflikte: Problem und Konfliktlösung, Verbesserung der Kommunikation, Mitarbeiterbindung

- Team: Konflikt- und Beziehungsklärung, Verbesserung von Arbeitsabläufen

- Klagemauer: Entlastung, Burn-out-Prophylaxe

- Weiterbildung: Supervision mit fachlichem Input

Kann und soll Supervision nicht alle diese Themen abdecken? Grundsätzlich ja, allerdings lassen sich drei gewichtige Argumente gegen »Gießkannenaufträge « an externe Berater anführen:

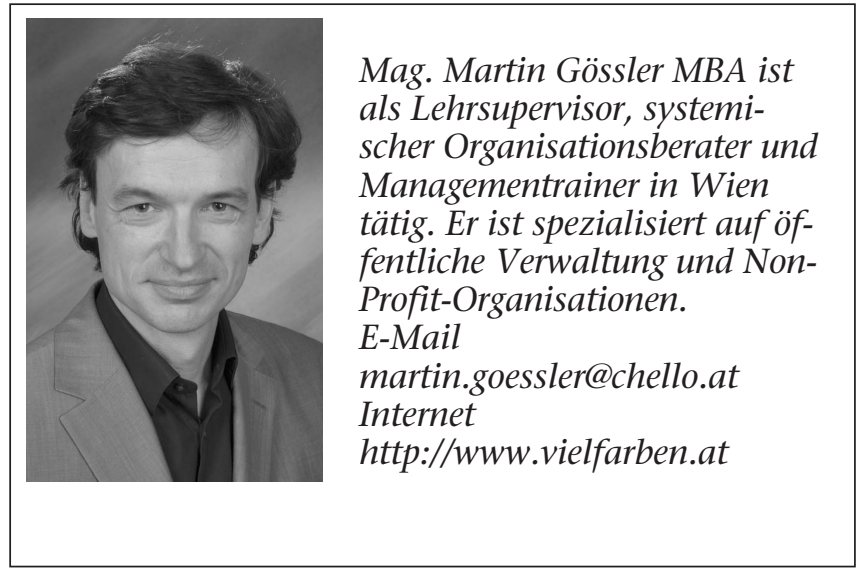

- In Zeiten knapper Ressourcen soll Supervision dort eingesetzt werden, wo die größte Wirkung zu erwarten ist.

- Manchmal führen andere Wege besser zum Ziel, beispielsweise Intervision statt Fallsupervision, eine Verbesserung der internen Kommunikation statt Teamentwicklung.

- Erst die Spezifizierung der Ziele macht eine präzise Auswahl der Berater möglich.

\section{Hebel 2: Horizontale Integration}

In den letzten Jahrzehnten haben wir einen beispiellosen Boom neuartiger Instrumente der Personalentwicklung und des Qualitätsmanagements erlebt. Oft stehen diese im Organisationsalltag unverknüpft nebeneinander, es fehlt an horizontaler Integration. Hier bieten sich viele Chancen für Synergien. Beispiele: Die Implementierung eines neuen Mitarbeiterarbeitergesprächs mit Supervision zu unterstützen. Oder die Umsetzung eines Qualitätsmanagementsystems mit Supervision zu gewährleisten.

\section{Hebel 3: Sorgfältige Auswahl}

Anders als in der Personalauswahl scheuen sich Verantwortliche manchmal bei der Auswahl von Beratern ebenso strenge Kriterien anzulegen. Selten gibt es ein Anforderungsprofil, nicht immer werden kritische Fragen gestellt. Dabei macht es Sinn, genau auszuwählen. Ebenso wie schlechte Klempner können auch schlechte Berater eine Sintflut hinterlassen. Anhand einiger Kriterien könnten Supervisoren passgenau ausgewählt werden (vgl. Kasten Seite 32). 


\begin{tabular}{|lll|}
\hline Anforderungsprofil für Supervisoren & \multicolumn{1}{c|}{$\begin{array}{l}\text { Relevanz des } \\
\text { Kriteriums für unsere } \\
\text { Organisation (1-5) }\end{array}$} & $\begin{array}{l}\text { Erfüllung des } \\
\text { Kriteriums (1-5) }\end{array}$
\end{tabular}

\section{Zukunftsfähigkeit sichern}

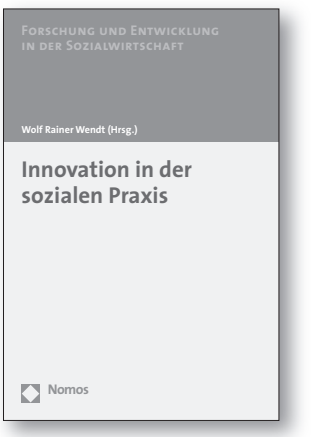

Innovation in der sozialen Praxis

Herausgegeben von Prof. Dr. Wolf Rainer Wendt, Universität Tübingen

2005,145 S., brosch., $27,-€$, ISBN 978-3-8329-1514-8 (Forschung und Entwicklung in der Sozialwirtschaft, $B d .4$ )

In den Beiträgen dieses Bandes wird die Frage behandelt, welche Bedeutung Innovationen im Wandel sozialer Verhältnisse und bei der Neustrukturierung sozialberuflicher Arbeit zukommt. In einem innovationstheoretischen Beitrag wird das Verständnis von Innovation erörtert. Innovationsprozesse in einzelnen Gebieten Sozialer Arbeit und den Ebenen der organisierten sozialen Versorgung sind Gegenstand von sechs weiteren Beiträgen aus der Praxis.

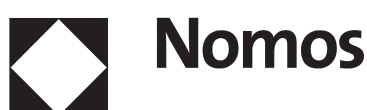

\section{Zehn Auswahlfragen an Supervisoren}

1. Wie viel Erfahrung und welches Wissen haben Sie in unserem Arbeitsfeld?

2. Welche beruflichen Erfahrungen haben Sie vor Ihrer Beratertätigkeit gemacht?

3. Wie viele ähnlich gelagerte Supervisionen haben Sie bereits gemacht?

3. Wo sehen Sie die wichtigsten Herausforderungen in unserem Arbeitsfeld in der Zukunft?

5. Können Sie uns in fünf Sätzen sagen, was der Vorteil Ihres Beratungsansatzes ist? In welchen Fällen halten Sie Ihren Ansatz für nicht geeignet?

6. Was sind Ihre drei besten, was Ihre drei schlechtesten Eigenschaften als Berater?

7. Angenommen, wir würden Ihrer früheren Supervisionskunden fragen, was würden diese uns über Sie erzählen?

8. Was wäre für Sie selbst ein gutes Ergebnis dieser Supervision?

9. Was ist Ihr Honorar und was genau ist darin enthalten?

10. Angenommen es passiert $\mathrm{x}$ (eine für die Supervision mögliche und herausfordernde Situation schildern) was würden Sie tun? 


\section{Hebel 4: Positives Image kreieren}

Ein häufiges Szenario: Teams, denen viel Kompetenz zugeschrieben wird, beanspruchen Supervision. Andere weigern Supervision. Auch Supervision braucht internes Marketing. Hier einige Ideen aus der Praxis:

- Infoveranstaltungen mit Supervisoren und Supervisionsfans aus der Zielgruppe

- Supervisorenmesse: Supervisoren stellen sich vor Teams, denen nachgesagt wird »sie hätten es nötig «, ver-

ge, dass das Management kaum etwas darüber erfährt? Negative Konsequenzen: Wenig Qualitätssicherung in der Beraterauswahl, schwache Steuerung der Beratungsziele, wenig Nutzung der Supervisionsergebnisse für die Organisation. Supervision läuft Gefahr, zur Subversion zu mutieren.

Modell Amtsschimmel: In einem Fall war ich als Supervisor mit einem 15-stufigen Supervisionsprozess (von Beauftragung bis Rechnungslegung) konfrontiert, der von der sicherlich wohlmeinenden Personalabteilung entworfen wurde. Negative Konsequenzen: Teure Verwaltungsabläufe, demotivierende Wirkung auf interne Supervisionskunden, informelle Bypassregelungen und dadurch Glaubwürdigkeitsverlust der Personalabteilung.

Die Alternative: Serviceorientierte Prozesse in gemischten Arbeitsgruppen (Personal, Führungskräfte, Zielgruppenvertretern) entwerfen. Wenige, dafür aber lebbare formale Abläufe definieren. Und nach einiger Zeit gemeinsam beobachten, welche Folgen und nicht intendierte Nebenwirkungen diese Regelungen haben.

\section{Hebel 7: Supervision evaluieren}

Ein Workshop mit einer Altenhilfeorganisation mit 600 Mitarbeiterinnen und Mitarbeiter. Es ging um die Kosten von Supervision: »200.000 Euro jährlich inklusive der anteiligen Personalkosten der Teilnehmenden « meinte die Personalleiterin. Die Stirn des Geschäftsführers furchte sich blitzartig. Häuften sich doch kritische Meldungen über die zielgerichtete Verwendung dieses Budgets. Die Folge: Ein Projektteam bekam die Aufgabe, den Einsatz von Supervision zu evaluieren und Verbesserungsvorschläge zu erarbeiten. Kernfragen eines solchen Evaluationsprozesses:

- Was läuft bezüglich des Einsatzes von Supervision im Vergleich zu anderen Trägern besonders gut, was besonders schlecht?

- Welche Wirkungen erzielt Supervision? Wie wirkt sich Supervision auf die Betreuungsqualität aus?

- Was sind die wichtigsten »Lessons learned « aus gescheiterten Supervisionsprozessen?

- Verbesserungspotenzial: Wie kann die Effizienz und Effektivität von Supervision gesteigert werden?

\section{Schlussbemerkung} rund um Beraterauswahl, Beauftragung, Berichtswesen und Rechnungslegung entwickelt. Der Umgang mit externen Beratern hat sich in den letzten Jahren stark professionalisiert. Zwei dysfunktionale Modelle sind trotzdem noch oft anzutreffen: Das Modell »Undercover « und das Modell »Amtsschimmel $\ll$.

Modell Undercover: Supervisoren werden auf eher informellem Weg ausgewählt und beauftragt. Die Wichtigkeit von Vertraulichkeit wird oft betont. Es gibt nur wenige kommunikative Schnittstellen zur Organisation. Eine in der Beraterwelt einzigartige Konstellation: Wäre es in einer Gummibärchenfabrik vorstellbar, dass Teams direkt Berater beauftragen, um mit diesen über das Gummibärchenmachen zu reflektieren — allerdings unter der Aufla-
Ein Trend ist in Wirtschaft, Verwaltung und Non-ProfitSektor deutlich beobachtbar: Der Einkauf und Einsatz von Beratungsleistungen wird professionalisiert. Eine erfreuliche Entwicklung. Denn richtig eingesetzte, gute Beratung ist ein hochwirksames Mittel zur Entwicklung fachlicher und organisationaler Professionalität. 